\title{
1 Einleitung: Spanisch im mehrsprachigen Berlin
}

Wie die meisten großen Städte Deutschlands und Europas ist Berlin eine ausgeprägt mehrsprachige Stadt. Den Fokus auf das Spanische in Berlin als eine einzelne Sprache zu legen mag zunächst sehr einschränkend erscheinen. Ein Gesamtbild zu zeichnen ist jedoch logischerweise gar nicht das Ziel dieses Buches. Vielmehr geht es darum, die Stellung des Spanischen im mehrsprachigen Gefüge der Stadt besser einschätzen zu können. Interessant ist das Spanische unter anderem deshalb, weil es im Vergleich zu anderen bedeutsamen Sprachen eine Art Zwischenstellung hat: Es wird relativ häufig in der Schule gelernt, gilt aber bei zunehmendem Interesse noch immer als weniger kanonisch als beispielsweise Englisch oder auch Französisch (Ministerio de educación y ciencia 2005: 17, 25; Bär 2017; Hoffmann/Malecki 2018: 20-21). ${ }^{1}$ Es ist zudem durchaus verbunden mit Migration, gilt aber als weitaus weniger emblematisch ,migrantisch als etwa Türkisch oder Arabisch. Für das Fallbeispiel Hamburg etwa ordnet Redder (2013: 263-264) das Spanische gleichzeitig zwei unterschiedlichen Segmenten der städtischen Mehrsprachigkeit zu, nämlich der Kategorie „languages spoken by migrants from countries frequently visited for touristic purposes" und der Kategorie „languages spoken by migrants that are also taught in schools“. Spanisch ist daher ein besonders faszinierender Mosaikstein im enorm diversen Sprachengefüge Berlins. Berlin ist dabei eher als exemplarisch zu sehen und nicht unbedingt als Sonderfall. Die meisten Befunde zur Bedeutung des Spanischen dürften sich zumindest auf andere größere Städte in Deutschland übertragen lassen, wenn sie nicht gar eine breitere Gültigkeit haben. So haben beispielsweise München und Hamburg vergleichbare Zahlen von Besuchenden und Einwohner`innen aus spanischsprachigen Ländern (Krämer 2019b: 248). ${ }^{2}$ Bei der Position von Spanisch als Fremdsprache in den Schulen zeigen sich in Deutschland insbesondere Ähnlichkeiten bei den Stadtstaaten (Hoffmann/Malecki 2018: 20-21). ${ }^{3}$

1 Kapitel 5 geht ausführlicher auf die Stellung des Spanischen im Berliner Schulsystem ein und zeigt Vergleiche zur Lage in anderen Teilen Deutschlands.

2 In den Kapiteln 4 und 9 wird die Aussagekraft der Zahlen aus Bevölkerungs- und Tourismusstatistiken näher diskutiert.

3 Auch in der Schweiz lassen sich deutliche Parallelen finden: Werlen (2010: 51) zeigt beispielsweise auf Basis repräsentativer Daten aus dem Jahr 2006, dass in allen Sprachgebieten der Schweiz gut 10\% der Bevölkerung Fremdsprachenkenntnisse des Spanischen hatten. Es ist damit nach dem Englischen die am zweithäufigsten gelernte Sprache, die nicht zugleich Landessprache der Schweiz ist.

Ә Open Access. () 2020 Philipp Krämer, publiziert von De Gruyter. (cc)BY Dieses Werk ist lizenziert unter der Creative Commons Attribution 4.0 International Lizenz. 
Welche Stellung das Spanische unter anderen Sprachen in Berlin einnimmt, soll mit diesem Buch genauer ausgelotet werden. Verschiedene Dimensionen stehen dabei im Mittelpunkt: Es soll gefragt werden, welche Rolle das Spanische im Bildungswesen in Berlin spielt, welche Einstellungen und Meinungen mit der Sprache verbunden sind, welche Motivationen und Erwartungen diejenigen haben, die es lernen. Dabei gerät auch die ökonomische Bedeutung des Spanischen ins Blickfeld, also die Frage, inwieweit es mit beruflichen bzw. materiellen Perspektiven in Verbindung gebracht wird und welches Gewicht diese Dimension gegenüber anderen Faktoren wie kommunikativer Reichweite, interkulturellem Interesse oder privaten Verbindungen einnimmt. Mit anderen Worten: Welche Erwartungen, Vorstellungen oder Hoffnungen werden an das Spanische geknüpft?

Der Fokus wird bei all diesen Fragen auf dem Spanischen als Fremdsprache liegen. Nicht nur in Deutschland, sondern in vielen Regionen der Welt ist Spanisch eine beliebte Sprache, die immer häufiger auch als Zweit- und Fremdsprache erworben wird (Klump/Willems 2012: 164; Pastor Cesteros 2016: 42). Die Beliebtheit des Spanischen dürfte dabei auch mit einem Schneeballeffekt zu erklären sein, wie Coulmas (1992: 80) ihn zeigt: „The more people learn a language, the more useful it becomes, and the more useful it is, the more people want to learn it.“

Kapitel 4 wirft einen kurzen Blick auf die Präsenz des Spanischen als Erstsprache in Berlin, weil dies selbstverständlich auch einen Einfluss auf die Einschätzung der Sprache durch L2-Sprecher^innen hat. Danach richtet sich die Aufmerksamkeit stärker auf diejenigen, die das Spanische erlernen und die damit bewusst in einen größeren Zusammenhang dessen eintreten, was als Global Spanish bezeichnet wird. Hier gilt es zu berücksichtigen, dass das Spanische - wie auch andere Fremdsprachen - von unterschiedlichen Gruppen aus unterschiedlichen Motiven und mit unterschiedlichen Zielen gelernt wird. Im Mittelpunkt stehen deshalb Befragungsstudien mit vier Gruppen: Studierende des Spanischen an der Freien Universität Berlin, Studierende anderer Fächer, die einen Spanischkurs an der Technischen Universität Berlin belegen, Teilnehmerinnen an Spanischkursen von Berliner Volkshochschulen und Beschäftigte im Berliner Gastgewerbe mit oder ohne Spanischkenntnisse.

Ob das Spanische beispielsweise tatsächlich an Nutzen gewinnt, wie die oben zitierte Passage von Coulmas nahelegen müsste, ${ }^{4}$ und welchen Nutzen das

\footnotetext{
4 In den Eurobarometer-Umfragen von 1995 bis 2005 stieg der Anteil derjenigen, die Spanisch als nützlich Sprache einschätzten, in den ,alten' EU-Mitgliedsstaaten leicht an: von 16 auf 21 Prozent. In den ,neuen` Mitgliedsstaaten blieb der Wert unverändert niedrig bei 3 Prozent (Pietiläinen 2011: 6). Auch bis 2012 hatte sich der Wert mit 14 Prozent für die Gesamt-EU wenig verändert (EU-Kommission 2012: 69; 141). Neuere Eurobarometer-Ergebnisse zeigen besonders in der jüngeren Generation eine enorm hohe Beliebtheit des Spanischen, die jedoch in der Befragung nicht
} 
Spanische in den Augen der Lernenden hat, bleibt zu zeigen. Wird nach der Bedeutung einer Sprache gefragt, so muss man stets berücksichtigen, dass dies eine Frage der Perspektive ist: „,Bedeutung haben“ impliziert auch ein ,für jemanden“ oder ,für etwas““ (Schnitzer 2012: 157). Dieses Buch soll daher einen Beitrag dazu liefern, die Motivationen und Spracheinstellungen der vier genannten Zielgruppen genauer zu beleuchten, für die das Spanische eine wichtige Bedeutung zu haben scheint. Welche das jeweils ist, kann mit Hilfe der Befragungsstudien genauer ausgelotet werden.

mehr an Nutzen geknüpft war. Im Jahr 2018 lag Spanisch mit 35 Prozent auf Platz 1 der Sprachen, die junge Europäer^innen im Alter von 15 bis 30 Jahren gerne lernen möchten. Bei den Befragten aus Deutschland war der Wert mit 44 Prozent noch höher (EU-Kommission 2018: 54-56). 
